\title{
Effects of Blade Number on Hydraulic Force Perturbation on Impeller of Volute-Type Centrifugal Pump*
}

\author{
Yutaka HASEGAWA**, Koji KIKUYAMA** \\ and Takao MAEDA**
}

\begin{abstract}
Perturbations of the hydrodynamic force on a three-blade centrifugal pump impeller were analyzed by transforming the static pressure change obtained on an impeller blade into Fourier series. Comparisons of the forces exerted on a blade and an impeller were made between the three- and seven-blade impellers, and the effects of the blade number on the hydrodynamic force were examined. It is found that there are few differences in the hydrodynamic force exerted on one blade between the impellers of different blade numbers. However, the hydrodynamic force on the impeller obtained by the vector summation of each blade force exhibits larger fluctuations for the three-blade impeller than that for the seven-blade one.
\end{abstract}

Key Words: Fluid Machinery, Fluctuating Pressure, Fluid Force, Centrifugal Pump, Volute Casing, Blade Number

\section{Introduction}

In order to design centrifugal pumps with high speed and high reliability, it is a most important problem to suppress the fluctuating hydrodynamic force on the pump impeller. Some studies have been made on the perturbation of the radial force.

Kawata et al. ${ }^{(1)}$ measured the fluctuating components of the radial force of centrifugal pumps with 6 and 7 blades, and Yamaguchi and Ito ${ }^{(2)}$ measured fluctuating hydrodynamic forces exerted on a pump-turbine impeller in normal running and transient operating conditions. Aoki ${ }^{(3)}$ measured the pressure inside the impeller passage of a one-blade centrifugal pump and clarified the relationship between pressure distribution and radial thrust. The radial thrust on two-dimensional and quasi-three-dimensional impellers has been calculated theoretically using a singularity meth$\mathrm{od}^{(4) \sim(6)}$. In the previous paper, the present authors examined the periodically fluctuating force on a

* Received 19th March, 1990. Paper No. 88-0981A

** Faculty of Engineering, Nagoya University, Furocho, Chikusa-ku, Nagoya 464-01, Japan seven-blade impeller and transformed it into the sum of the components having a frequency of blade number times rotational speed and its harmonic frequencies $^{(7)}$.

For impellers with a reduced blade number, which are sometimes used for air-water or solid-water mixtures, the blade load is increased and the interference of the impeller exit flow with the volute tongue generates large fluctuating forces on the impeller. In this paper, the fluctuating force on the three-blade impeller was examined experimentally and comparison was made with that of the seven-blade impeller.

\section{Nomenclature}

$A_{2}$ : impeller exit area including thickness of blades

$C_{j}:$ Fourier coefficient of phase-lock averaged pressure

$F:$ hydrodynamic force vector

$p:$ measured static pressure

$Q:$ discharge flow rate of pump

$\boldsymbol{R}, R$ : vector of dimensionless hydrodynamic force and its magnitude

$\boldsymbol{R}=\boldsymbol{F} /\left(\rho A_{2} \boldsymbol{u}_{2}^{2}\right)$ 
$r, r_{2}:$ radii of measuring point and impeller exit

$T:$ moment of hydrodynamic force about the pump axis

$u, u_{2}:$ circumferential velocities of measuring point and impeller exit

$Z$ : blade number

$\alpha:$ angle of time mean radial thrust

$\theta:$ circumferential position of blade

$\nu$ : coefficient of moment of hydrodynamic force

$=T /\left(\rho A_{2} u_{2}^{2} r_{2}\right)$

$\xi_{j}$ : phase of the $j$-th term in Fourier series of phase-lock averaged pressure

$\rho:$ density of water

$\phi:$ discharge coefficient $=Q /\left(A_{2} u_{2}\right)$

suffixes and operators

$b$ : blade

$i$ : impeller

$s:$ measuring point in suction pipe

$2:$ just downstream of impeller exit

$-:$ time mean value

$\Delta:$ fluctuating term

\section{Equipment and Method of Experiment}

The impeller used in this experiment is shown in Fig. 1. The configurations of the blades both for the three-and seven-blade impellers are the same as those used in the previous paper ${ }^{(7)}$. The location of the pressure tappings inside the impeller and volute is shown in the same figure. Eighteen tappings were drilled in the blade surface at a height of $15 \mathrm{~mm}$ from the hub surface, and twelve tappings were also drilled in the hub. The static pressures in the volute were measured in twelve tappings located on the basic circle of the volute casing of a radius $r_{v}=185 \mathrm{~mm}$, as

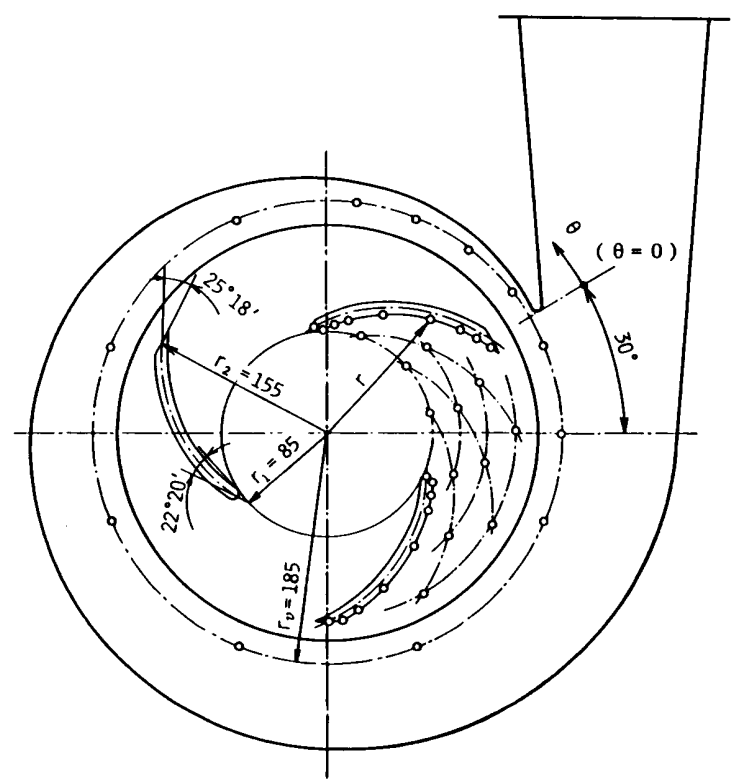

Fig. 1 Configurations of impeller blade and volute casing shown in Fig. 1. In order to indicate the circumferential position of the pressure tappings, the angle is taken as that of the measuring blade from the leading edge of the volute tongue. The rotational speed of the pump was set at $595 \mathrm{~min}^{-1}$ and the flow rates of the pump were taken as $\phi=0.032,0.064,0.096,0.101,0.117$, 0.128 , and 0.160 .

The rate of the increase in the cross-sectional area of the volute is constant and approximately 9.66 $\mathrm{cm}^{2} / \mathrm{rad}$. The clearence between the impeller outlet and the volute tongue is set to be $37 \mathrm{~mm}$.

Figure 2 shows the total pressure head of the pumps for $Z=3$ and 7 , in which the decrease in the blade number from seven to three is seen to cause the decrease in the pump head. In the figure, Busemann's theoretical impeller heads ${ }^{(8)}$ are shown both for $Z=3$ and 7 , and the characteristic line of the volute is also plotted. From the intersection of the curves, the optimum operation points of the pumps are seen to be $\phi_{\text {opt }}$ $=0.074$ and 0.094 for $Z=3$ and 7 , respectively.

The static pressures measured in the rotating system were transmitted to the stationary system by means of slip rings, sampled during 256 impeller rotations and processed by a microcomputer into the phase-lock averaged and fluctuating components at the measuring points of every $(360 / 252)$ degree. Let $p_{m}(\phi, \theta)$ be the instantaneous pressure in the circum. ferential point $\theta$ of the $m$-th rotation; it is separated into the time mean, and both the periodically and nonperiodically fluctuating components, as expressed by the following equation,

$$
\begin{aligned}
& p_{m}(\phi, \theta)=P(\phi, \theta)+p_{m}^{\prime}(\phi, \theta) \\
& \quad=\bar{P}(\phi)+[P(\phi, \theta)-\bar{P}(\phi)]+p_{m}^{\prime}(\phi, \theta),
\end{aligned}
$$

where $P(\phi, \theta)$ is the phase-lock mean pressure defined by the following equation as

$$
P(\phi, \theta)=(1 / M) \sum_{m=1}^{M} p_{m}(\phi, \theta) \text {. }
$$

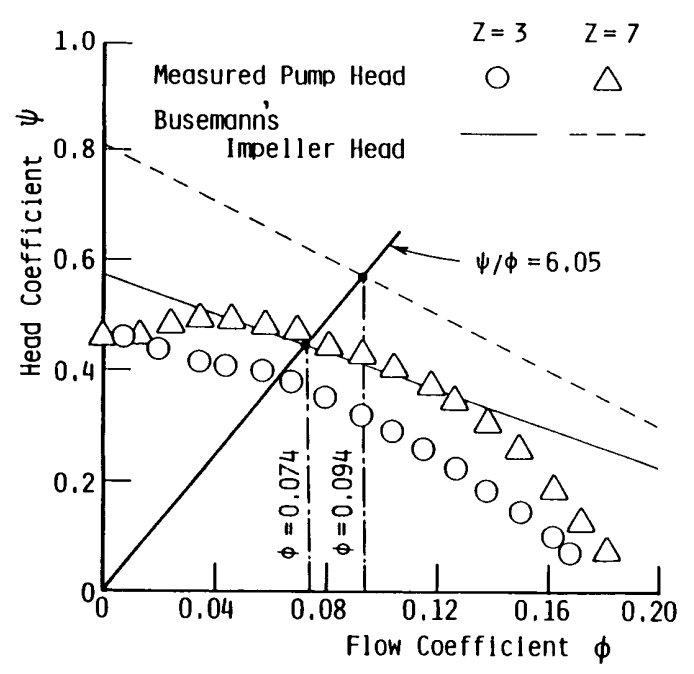

Fig. 2 Characteristic curve of pump 
The time mean value of $\bar{P}(\phi)$ is

$$
\bar{P}(\phi)=(1 / 2 \pi) \int_{0}^{2 \pi} P(\phi, \theta) d \theta .
$$

The strength of the nonperiodic fluctuating component $p_{m}^{\prime}(\phi, \theta)$ is evaluated by its rms value as

$$
p^{\prime}(\phi, \theta)=\sqrt{(1 / M) \sum_{m=1}^{M}\left[p_{m}(\phi, \theta)-P(\phi, \theta)\right]^{2}} .
$$

The pressure components defined by the above equations are made dimensionless, as given below by the pressure coefficients :

$$
\begin{aligned}
& C_{p}(\phi, \theta)=\left[P(\phi, \theta)-\bar{P}_{s}\right] /\left(\rho u_{2}^{2} / 2\right) \\
& \bar{C}_{p}(\phi)=\left[\bar{P}(\phi)-\bar{P}_{s}\right] /\left(\rho u_{2}^{2} / 2\right) \\
& C_{p}^{\prime}(\phi, \theta)=P^{\prime}(\phi, \theta) /\left(\rho u_{2}^{2} / 2\right) \\
& \bar{C}_{p}^{\prime}(\phi)=(1 / 2 \pi) \int_{0}^{2 \pi} C_{p}^{\prime}(\phi, \theta) d \theta,
\end{aligned}
$$

where $\bar{P}_{s}$ and $u_{2}$ denote the time-mean suction pressure and the peripheral speed of the impeller outlet, respectively. The phase-lock mean pressure coefficients $C_{p}(\phi, \theta)$ are also transformed into Fourier series as

$$
C_{p}(\phi, \theta)=\bar{C}_{p}(\phi)+\sum_{j=1}^{\infty} C_{j}(\phi) \sin \left(j \theta+\xi_{j}\right),
$$

where $C_{j}$ and $\xi_{j}$ are the amplitude and the phase difference in the $j$-th term of the periodic pressure fluctuation.

\section{Experimental Results and Discussions}

\section{1 Pressure fluctuations}

As the pressure inside the impeller channel changes periodically in accordance with the pressure distribution in the volute, the circumferential changes of the static pressure are compared between the volute and at the impeller exit in Fig. 3, for the flow rates of 0.032 and 0.160 of three- and seven-blade pumps. The pressures in the volute of the seven-blade pump exhibit larger values than those at the impeller exit due to the divergence of flow between the impeller and the volute. With the three-blade pump, how- ever, no increase in the pressure can be seen between the impeller exit and the volute due to the hydraulic losses caused by the flow mixing downstream of the impeller exit. There are few differences in the pressure change of the impeller exit between $Z=3$ and 7 , and the pressures in the volute also exhibit almost the same tendency. In the lower flow rate of $\phi=0.032$, a large change in pressure is seen to be limited to a narrow region near the volute tongue $(\theta=0)$, but at the flow rate of $\phi=0.160$, the pressure change extends over the circumference of the exit.

Figures 4 and 5 show the contours of equivalue in the phase-lock mean pressure $C_{p}(\phi, \theta)$ inside the impeller at every $(2 / 3) \pi$ radisn of the impeller rotation. When the flow rate is equal to 0.032 , the contours exhibit almost coaxial circles showing that the pressure increase is dominated by the centrifugal force of the impeller rotation. Corresponding to the circumferentially uneven pressure distribution inside the volute, the pressure in the impeller channel changes with the

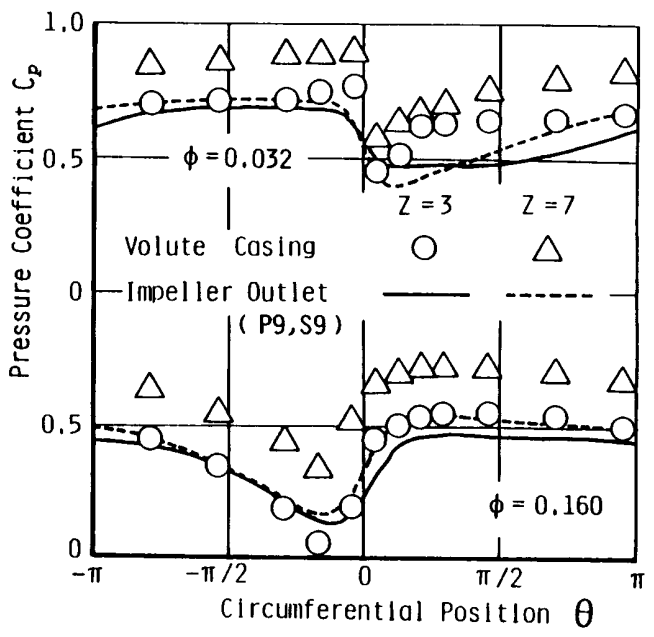

Fig. 3 Circumferential change of pressure at impeller exit and in volute casing
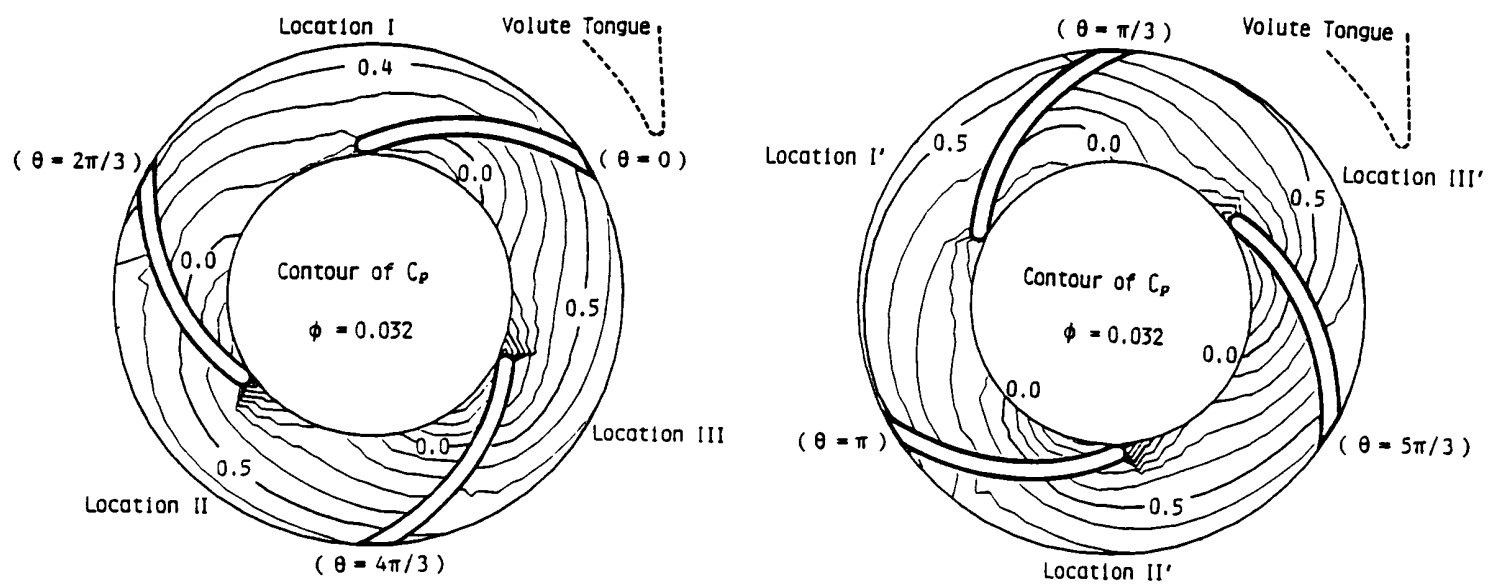

Fig. 4 Distribution of pressure in impeller channel $(\phi=0.032)$ 

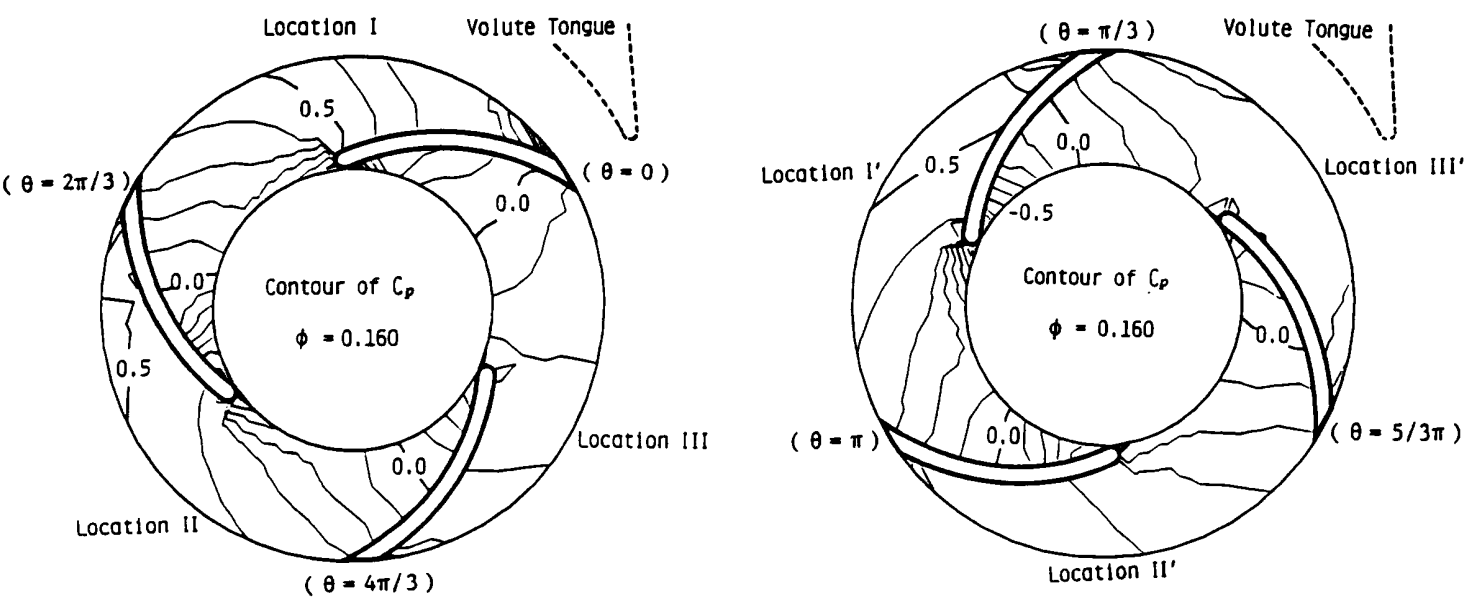

Fig. 5 Distribution of pressure in impeller channel $(\phi=0.160)$

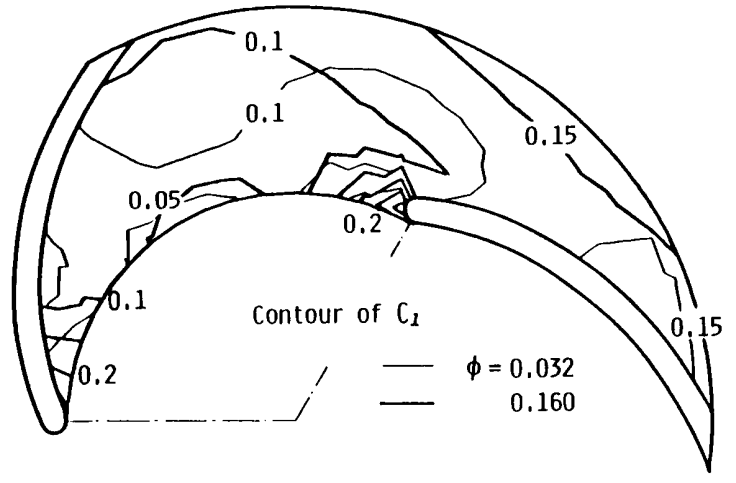

Fig. 6 Distribution of Fourier coefficient of $C_{1}$

rotation, being decreased at location I in Fig. 4. The pressure drop inside this impeller channel brings about the increase in the inflow to the channel, which can be assumed by a large pressure gradient near the leading edge of the blade at the circumferential positions of $\theta$ $=(2 / 3) \pi$, and $(4 / 3) \pi$.

When the flow rate is increased to $\phi=0.160$, the pressure difference between the suction and pressure surfaces is increased and the equipressure curves tend to be normal to the blade surfaces. In this case, the configuration of the pressure curves changes largely with the rotation, and the pressure gradient decreases at impeller locations of III and III'.

Figure 6 shows the distribution of the coefficient in the Fourier series, $C_{1}$ in Eq. (6), which corresponds to the intensity of the periodic fluctuation in the channel. The value of $C_{1}$, takes its maximum near the leading edge of the blade due to the circumferential variation of the impeller inflow, as described above.

Figure 7 shows the distribution of the intensity of the non-periodically fluctuating pressure component, $\bar{C}_{p}^{\prime}(\phi)$, which is obtained by the circumferential aver-

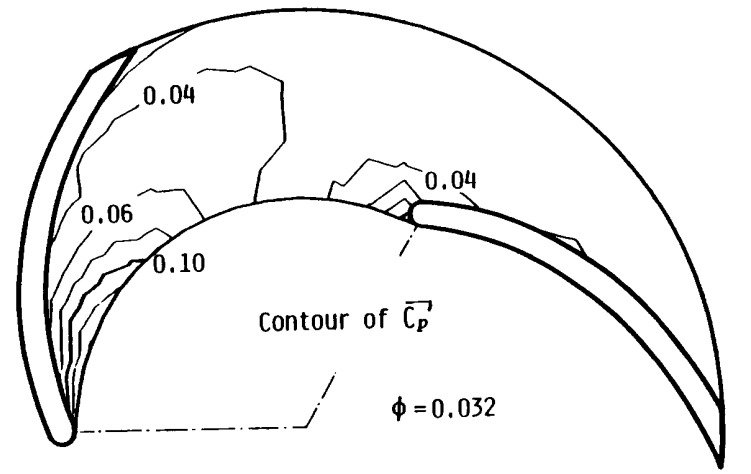

Fig. 7 Distribution of rms value of nonperiodic fluctuation

age of $C_{p}^{\prime}(\phi, \theta)$ defined by Eq. ( 5$)$. At the flow rate of $\phi=0.032$, the flow separates from the blade suction surface near the impeller inlet, causing a larger fluctuation in the pressure there. The increase in the nonperiodic pressure fluctuation in the suction region of the channel inlet may be brought about by the nonsteady occurrence of vortex shedding and shifts of the separation point.

\subsection{Hydrodynamic forces on blades and impel- lers}

By integrating the phase-lock averaged pressure load and its moment around the pump axis over the blade surface, the periodic change of the force and the moment exerted on a blade as well as on the whole impeller can be calculated. Figure 8 shows the changes of the dimensionless components of the fluctuating moment on one blade, $\nu_{b}\left(=T_{b} / \rho A_{2} u_{2}^{2} r_{2}\right)$, for the three- and seven-blade impellers. Although the magnitude of the moment is larger for the three-blade impeller, similar changes in $\nu_{b}$ can be seen between the impellers of different blade numbers. When the pump is operated near the optimum flow rate of $\phi=$ 0.096 , the change in the moment due to a blade load is 
flattened during rotation but in off-design flow conditions, $\nu_{b}$ shows a larger change and takes its maximum or minimum when the blade passes by the volute tongue.

By summing up the moments on all blades, the total moment exerted on the impeller can be obtained as follows.

Let the moment of the $k$-th blade, $\nu_{b}^{k}$, be given by the following Fourier series,

$$
\nu_{b}^{k}=\left(\nu_{b}\right)_{0}+\sum_{j=1}^{\infty}\left(\nu_{b}\right)_{j} \sin \left[j(\theta+2 \pi k / Z)+\xi_{j}^{\prime}\right],
$$

where $\left(\nu_{b}\right)_{0}$ and $\left(\nu_{b}\right)_{j}$ denote the Fourier coefficients of the 0 -th and $j$-th terms, respectively, $\xi_{j}^{\prime}$ being the phase difference of the $j$-th term. The total moment on the impeller, $\nu_{i}$, is given by

$$
\nu_{i}=\sum_{k=0}^{z-1} \nu_{b}^{k}
$$

By the substitution of Eq. ( 7 ), the total moment of the impeller given by the above equation is found to have only the terms of the $Z$-th component and its harmonics and is thus given as

$$
\begin{aligned}
\nu_{i} & =Z\left(\nu_{b}\right)_{0}+Z \sum_{j=1}^{\infty}\left\{\left(\nu_{b}\right)_{z j} \sin \left[Z j^{\prime} \theta+\xi^{\prime} Z j^{\prime}\right]\right\} \\
& =Z\left(\nu_{b}\right)_{0}+\Delta \nu_{i},
\end{aligned}
$$

where $j=j^{\prime} Z\left(j^{\prime}=1,2, \cdots\right)$. The second term of the above equation, $\Delta \nu_{i}$, denotes the fluctuating component of the moment on the impeller.

Figure 9 shows the changes in $\nu_{i}$ during the $1 / Z$ rotation of the impeller. The curves of $\nu_{i}$ exhibit sinusoidal changes because the cyclic change in $\nu_{i}$ is mainly dominated by the term $j^{\prime}=1$ of Eq. ( 9 ) and the oscillations of higher frequency die away due to the existing clearance between the volute tongue and the impeller exit. Compared with the seven-blade impeller, the perturbation of the moment for the impeller of

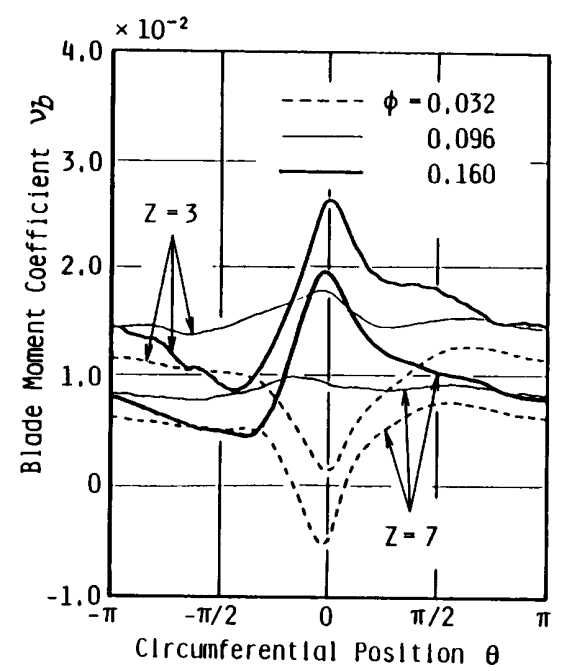

Fig. 8 Circumferential change in moment of hydrodynamic force on one blade
$Z=3$ is increased when the pump operates with the off-design of $\phi=0.032$ and 0.160 .

In order to show the cyclic change in the hydrodynamic force on a blade, the traces of the force vector were plotted as shown in Fig. 10. The origin of the vector was fixed at the radial distance of $(r$ $\left.-r_{1}\right) /\left(r_{2}-r_{1}\right)=0.4$. This is because the acting line of the force vector almost intersects with the blade center line $C-C^{\prime}$ (in Fig. 10) at this radial position for the different flow rates and the different blade positions. As shown in the figure, the hydrodynamic force acts in almost the same direction, but its magnitude changes during one impeller rotation, and a considerably large change can be seen when the blades pass by the volute tongue.

Let the fluctuating components of the force acting on a blade of $k=0$ be given in the rotating coordinates by the following equation

$$
\left(\boldsymbol{F}_{b}\right)^{0}=\sum_{j=1}^{\infty}\left[\boldsymbol{A}_{j} \exp (i j \theta)+\boldsymbol{B}_{j} \exp (-i j \theta)\right],
$$

where $i^{2}=-1$. The imaginary and real parts of Eq. (10) correspond to the orthogonal components of the fluctuating force acting in the rotating plane normal to the pump axis. $\boldsymbol{A}_{j}$ and $\boldsymbol{B}_{j}$ denote the vector coefficients of the $j$-th term in the Fourier series. Thus, the radial thrust on the impeller is given by the

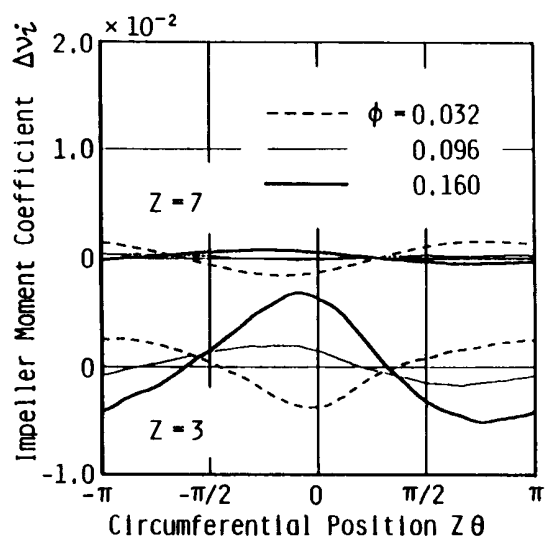

Fig. 9 Circumferential change in moment of hydrodynamic force on impeller

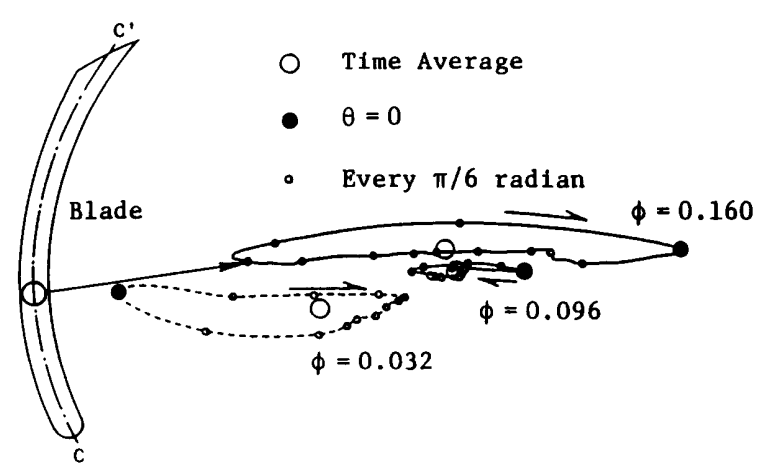

Fig. 10 Vectors of hydrodynamic force on a blade 


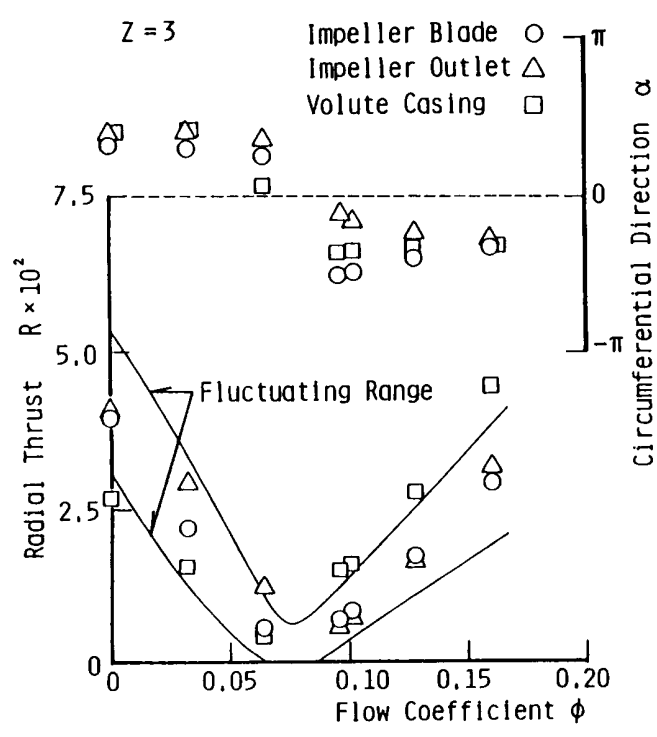

(a) $Z=3$

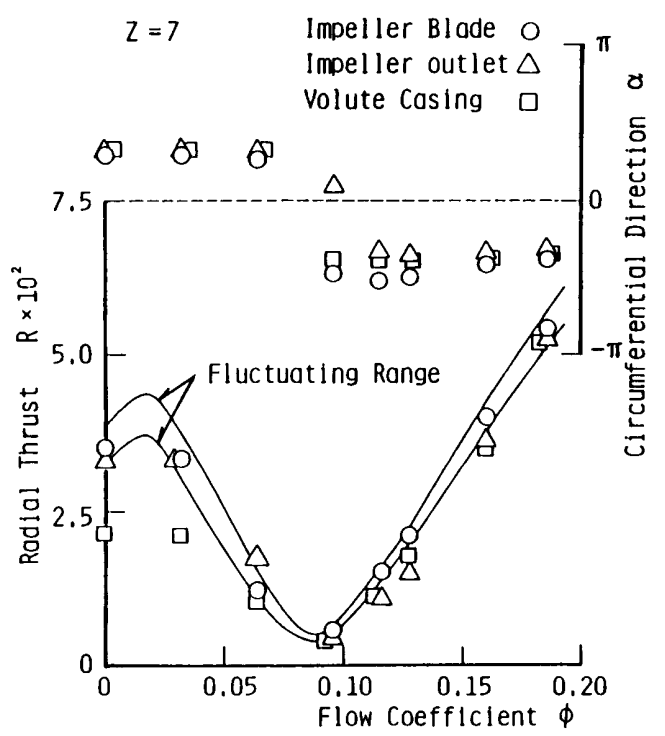

(b) $Z=7$

Fig. 11 Radial thrust and its fluctuating range

summation of forces $\left(\boldsymbol{F}_{b}\right)_{j}^{k}$ in the rotating coordinates as

$$
\begin{aligned}
\boldsymbol{F}_{i} & =Z \boldsymbol{A}_{1} \exp (i \theta)+Z \sum_{j^{\prime \prime 1}}^{\infty}\left\{\boldsymbol{A}_{\boldsymbol{z}=j^{\prime}+1} \exp \left[i\left(Z j^{\prime}+1\right) \theta\right]\right. \\
& \left.+\boldsymbol{B}_{\boldsymbol{z}^{\prime}-1} \exp \left[-i\left(Z j^{\prime}-1\right) \theta\right]\right\} .
\end{aligned}
$$

By transforming the above force into the stationary coordinates,

$$
\begin{aligned}
\boldsymbol{F}_{i}^{\prime} & =\boldsymbol{F}_{i} \exp (-i \theta) \\
& =\boldsymbol{Z} \boldsymbol{A}_{\mathbf{1}}+Z \sum_{j^{\prime}=1}^{\infty}\left[\boldsymbol{A}_{\boldsymbol{j}^{\prime}+1} \exp \left(i \boldsymbol{Z}^{\prime} \theta\right)\right. \\
& \left.+\boldsymbol{B}_{\boldsymbol{k}^{\prime}-1} \exp \left(-i Z j^{\prime} \theta\right)\right] .
\end{aligned}
$$

From the above equation, it is found that the steady component of the radial thrust in the stationary system corresponds to the first term of Eq. (11), having the same frequency as the impeller rotation. The periodically fluctuating components of the radial force correspond to the summation of the pressure loads having frequencies of $\left(z j^{\prime} \pm 1\right)$ times the impeller rotation in Eq. (10).

Figures 11(a) and (b) show the time mean magnitude, $R$, and the phase angle, $\alpha$, of the radial thrust for both $Z=3$ and 7 . Three kinds of data, obtained on the blade load, at the impeller exit, and in the volute, respectively, are plotted for comparison. From the figures, the forces from the blade load and the exit pressure change coincide well with each other when $\phi \geqq 0.10$, but diverges when $\phi \fallingdotseq 0.05$, possibly due to the complex three-dimensional flow pattern at the impeller inlet. Comparatively large discrepancies in $R$ for the impeller of $Z=3$ are attributed to the difference in the pressure distributions inside the volute and the impeller exit. The directions of the resultant radial thrusts obtained by the three methods are in good accordance with each other, as shown by the change of $\phi$. The radial thrust takes its minimum value at its optimum flow rates, shown in Fig. 2.

The comparison of the radial thrust between the three- and seven-blade impellers shows that the thrust of the seven-blade impeller exhibits larger values over the whole flow range. This is explained as follows.

As shown by Eq. (12), the time mean value of the hydrodynamic force is given by the blade number $Z$ times the Fourier coefficient $\boldsymbol{A}_{1}$. The latter denotes the magnitude of the cyclic change of the pressure load on a blade, and is considered to be the same magnitude for the two impellers from the changes in the moment of a blade both for $Z=3$ and 7 in Fig. 8 . Thus, the radial thrust on an impeller is larger for $Z$ $=7$ than that for $Z=3$.

The width of the fluctuation of the radial thrust in Fig. 11 increases as the flow rate departs from the optimal one and for the impeller of $Z=3$. Its magnitude attains 95 and 65 percent of the time mean value of when the flow rate is decreased and increased to $\phi$ $=0.032$ and 0.160 , respectively. These fluctuating components of thrust are seen to be largely dominated by the $(Z \pm 1)$ th terms of the Fourier series of the pressure load [Eq. (11)].

\section{Conclusions}

From the pressure measurement in the threeblade impeller pump and the comparison of the results with those of the seven-blade impeller pump, the following results are obtained.

(1) The cyclic pressure change inside the impel- 
ler channel is enhanced near the leading edge and near the channel exit at the off-design flow rate. Due to the existence of relatively large clearance between the volute and the impeller, the abrupt change in the volute pressure distribution near the tongue is slightly smoothed at the impeller channel exit.

(2) There is little difference in the fluctuating components of the hydrodynamic force exerted on one blade between $Z=3$ and 7 ; however, their summations on the impeller exhibit a large difference in their amplitude, and it takes a larger value on the impeller of $Z=3$.

\section{Acknowledgements}

The authors wish to express their appreciation to Prof. K. Minemura, for his useful suggestions and also to Mr. E. Asakura and Mr. H. Murakami for their support in the experiment.

\section{References}

(1) Kawata, Y., Kanki, H. and Kawakami, T., Experimental Research of the Radial Force on the Centrifugal Pumps (lst Report, Influence of the Impeller Vane Number and the Casing Type), Trans. Jpn. Soc. Mech. Eng., (in Japanese), Vol. 49, No. 437, C (1983), p. 31.

(2) Yamaguchi, Y. and Ito, H., Experimantal Research on Stress Fluctuations in Runner Blades of a High-Head Francis-Type Pump-Turbine, Trans. Jpn. Soc. Mech. Eng., (in Japanese), Vol. 47, No. 413, B (1981), p. 95.

(3) Aoki, M., Instantaneous Pressure Distribution between Blades and Fluctuating Radial Thrust in the Single Blade Centrifugal Pump,Trans. Jpn. Soc. Mech. Eng., (in Japanese), Vol. 50, No. 451, B (1984), p. 661

(4) Kamimoto, G., Ohshima, T., Mizutani, M. and Mizutani, H., Applications of Singularity Method in Turbomachinery, Proc. 10th Symp. of I. A. H. R., Tokyo, (1980), p. 659.

( 5 ) Tsujimoto, Y., Imaichi, K., Tomohiro, T. and Gotoo, M., An Analysis of Unsteady Torque on a Quasi-Three Dimensionsl Radial Impeller, Trans. Jpn. Soc. Mech. Eng., (in Japanese), Vol.50, No. 450, B(1984), p. 371.

(6) Imaichi, K., Tsujimoto, Y. and Yoshida, Y., A Two-dimensional Analysis of the Interaction Effects of Radial Impeller in Volute Casing, Trans. Proc. 10 th Symp. of I. A. H. R., Tokyo, (1980), p. 635.

(7) Kikuyama, K., Hasegawa, Y. and Maeda, T., Unsteady Pressure Distributions and Forces on the Impeller Blades of a Centrifugal Pump, Trans. Jpn. Soc. Mech. Eng., (in Japanese), Vol. 54, No. 504, B (1988), p. 2038.

(8) Busemann, A., Das Förderhöhenverhăltnis radialer Kreiselpumpen mit logarithmischspiralingen Schaufen, Ztschr. für Angewandte Math. und Mech., Band 8, Heft 5 (1928), p.372. 